\title{
Conserved Charges in the Principal Chiral Model on a Supergroup
}

\author{
B.H. Miller, \\ Department of Mathematics, University of York, \\ Heslington Lane, York YO10 5DD, UK \\ Email: bhm101@york.ac.uk
}

\begin{abstract}
The classical principal chiral model in $1+1$ dimensions with target space a compact Lie supergroup is investigated. It is shown how to construct a local conserved charge given an invariant tensor of the Lie superalgebra. We calculate the super-Poisson brackets of these currents and argue that they are finitely generated. We show how to derive an infinite number of local charges in involution. We demonstrate that these charges Poisson commute with the non-local charges of the model.
\end{abstract}

\section{Introduction}

Finding exact methods of solving string theory on AdS space, as motivated by the AdS/CFT correspondence, remains a difficult problem. There are two schemes for describing such a superstring. The NSR description gives a free action in a flat background but the existence of the $R R$ vertex operators introduces seemingly insurmountable difficulties. Alternatively there is the GS formalism in which the supersymmetry exists on the target manifold which is then described as a Lie supergroup or supercoset space.

It has proved possible to describe superstring theory on $A d S_{n} \times S^{n}$ as a coset space $G / H$ (for example, $A d S_{2} \times S^{2}$ is the bosonic subalgebra of $\frac{P S U(1,1 \mid 2)}{U(1) \times U(1)}$ [1, and $A d S_{5} \times S^{5}$ is the same for $\frac{P S U(2,2 \mid 4)}{S O(4,1) \times S O(5)}$ [2, 3, 4].) Although $G$ is Ricci flat in both these instances, the coset space is not. However, $H$ is the invariant locus of a $\mathbb{Z}_{4}$ automorphism, and this permits the introduction of a WZ-term to provide a quantum conformal theory. (Indeed [5, 6] have demonstrated quantum conformal invariance given a $\mathbb{Z}_{n}$ automorphism.) Similarly superstring theory on $A d S_{3} \times S^{3}$ is related to a sigma model on $P S U(1,1 \mid 2)$ [7. More general work has looked at sigma models on the supergroups $P S L(n \mid n)$ [] . 
All of these models contain an infinite number of local and non-local conserved charges. Their existence constrains the S-matrix and permits its exact computation. These charges have been studied in both bosonic and worldsheet supersymmetric principal chiral models (PCMs) [9], and for sigma models on symmetric spaces [10, 11].

The PCM on any Lie supergroup is classically conformal. In the quantum model, the one loop beta function is proportional to the dual Coxeter number $h^{\vee}$ [8, 12], and there are some superalgebras for which this vanishes (namely $p s u(n \mid n)$ and $o s p(2 n+2 \mid 2 n)$ ). The PCM on these supergroups will therefore be quantum conformal [8, 13]. However we do not expect conformal invariance to survive in the quantum model for general supergroups when the model becomes massive. This paper is a modest attempt at understanding the algebra of these models' local charges, with the hope that some insight could be given to the perhaps more physical models.

Ultimately we find that the classical PCM on a supergroup has an infinite number of charges in involution. These charges are formed from integrals of local conserved currents, each associated to an invariant of the Lie superalgebra. Only a finite number of these are independent, and from these invariants it is possible to construct all conserved local currents.

For the $s u(m \mid n)$ models we will construct a set of currents which give rise to commuting charges. The construction will fail for $m=n$, so we restrict ourselves to the massive cases. The situation is analogous for $\operatorname{osp}(m \mid 2 n)$, except now a family of currents exists, depending on a free parameter $\alpha$. For $o s p(2 m \mid 2 n)$ an additional invariant exists, the superpfaffian [14, and requiring that the superpfaffian charge commutes with the other local charges fixes the value of $\alpha$, but only when the model is massive.

These charges are conserved classically, but anomalies might arise in the quantum model. However we anticipate that integrability survives because we can use Goldschmidt and Witten's method of anomaly counting [15] to show that higher spin conservation laws still exist.

In addition to the local charges, there are non-local charges which form a Super-Yangian structure [16, 17, 18]. We shall show that these non-local charges are in involution with the local charges.

\section{$1.1 \quad$ Lie Superalgebras}

We begin with a basic introduction to superalgebras, and refer to the literature [19, 20, 21] for a more thorough review. Throughout this paper, letters $A, B, C, \ldots$ will denote both bosonic and fermionic indices. $a, b, c, \ldots$ will be 
used for bosonic indices, and $\alpha, \beta, \gamma, \ldots$ for fermionic indices.

We begin with an associative Grassmann algebra $\Lambda=\Lambda_{\overline{0}} \oplus \Lambda_{\overline{1}}$ with sufficiently many anticommuting generators, where $\Lambda_{\overline{0}}$ (resp. $\Lambda_{\overline{1}}$ ) consists of commuting (resp. anticommuting) elements. We have the product rule $\Lambda_{\bar{i}} \cdot \Lambda_{\bar{j}} \subset \Lambda_{\overline{i+j}}$. (Addition modulo 2 is left implicit here and throughout.)

Given a supermatrix $X=\left(\begin{array}{cc}A & B \\ C & D\end{array}\right)$ we define it to be even (odd) if $A, D \in$ $\Lambda_{\overline{0}}\left(\Lambda_{\overline{1}}\right)$ and $B, C \in \Lambda_{\overline{1}}\left(\Lambda_{\overline{0}}\right)$. We write $\operatorname{deg}(X)=0$ if it is even and $\operatorname{deg}(X)=1$ if odd. We can then define the supertrace as

$$
\operatorname{Str}(X)=\operatorname{Tr}(A)-(-1)^{\operatorname{deg}(X)} \operatorname{Tr}(D)
$$

the supertranspose as

$$
X^{S T}=\left(\begin{array}{cc}
A^{T} & -(-1)^{\operatorname{deg}(X)} B^{T} \\
(-1)^{\operatorname{deg}(X)} C^{T} & D^{T}
\end{array}\right)
$$

and if $X$ is even and invertible, the superdeterminant as

$$
\operatorname{sdet}(X)=\frac{\operatorname{det}\left(A-B D^{-1} C\right)}{\operatorname{det}(D)}=\frac{\operatorname{det}(A)}{\operatorname{det}\left(D-C A^{-1} B\right)} .
$$

These satisfy the important properties

$$
\begin{gathered}
(X Y)^{S T}=(-1)^{\operatorname{deg}(X) \operatorname{deg}(Y)} Y^{S T} X^{S T} \\
\operatorname{Str}(X Y)=(-1)^{\operatorname{deg}(X) \operatorname{deg}(Y)} \operatorname{Str}(Y X), \operatorname{Str}\left(X^{S T}\right)=\operatorname{Str}(X) \\
\operatorname{sdet}(X Y)=\operatorname{sdet}(X) \operatorname{sdet}(Y), \operatorname{sdet}\left(X^{S T}\right)=\operatorname{sdet}(X) \\
\operatorname{sdet}(\exp (X))=\exp (\operatorname{Str}(X))
\end{gathered}
$$

We consider a compact connnected supergroup $G$ [19], either $S U(m \mid n)$ or the compact subgroup of $O S p(m \mid 2 n)$ which is connected to the identity, satisfying

$$
\begin{array}{rll}
S U(m \mid n): & \operatorname{sdet} X=1 & X X^{\dagger}=1 \\
O S p(m \mid 2 n): & \operatorname{sdet}(X)=1 & X^{S T} H X=H
\end{array}
$$

where $H=\left(\begin{array}{cc}I & 0 \\ 0 & J\end{array}\right)$ for an symmetric $I$ and symplectic $J$. There exists a basis for which $I$ is the identity $\mathbb{I}_{m}$ and $J=\left(\begin{array}{cc}0 & \mathbb{I}_{n} \\ -\mathbb{I}_{n} & 0\end{array}\right)$.

The relation between a supergroup and its superalgebra is similar to the bosonic case. The supergroup $G$ associated to the superalgebra $\mathbf{g}$ is the 
exponential mapping of the even subsuperalgebra of the Grassmann envelope $\Lambda \otimes \mathbf{g}$.

$$
g=\exp \left(x_{A} T^{A}\right)=\exp \left(x_{a} T^{a}\right) \exp \left(x_{\alpha} T^{\alpha}\right)
$$

$T^{A}$ generate the Lie superalgebra $\mathbf{g}=\mathbf{g}_{\overline{0}} \oplus \mathbf{g}_{\overline{1}}$ corresponding to $G$ (where $T^{a}$ (respectively $T^{\alpha}$ ) generate $\mathbf{g}_{\overline{0}}$ (respectively $\mathbf{g}_{\overline{1}}$ ). These satisfy the supercommutation relationship

$$
\left[T^{A}, T^{B}\right]=T^{A} T^{B}-(-1)^{\eta_{A} \eta_{B}} T^{B} T^{A}=f^{A B} T^{C}
$$

for structure constants $f^{A B}{ }_{C}$. Note that the even subspace $\mathbf{g}_{\overline{0}}$ is itself a Lie algebra, whereas $\mathbf{g}_{\overline{1}}$ is a $\mathbf{g}_{\overline{0}}$-module.

We denote by $\eta_{A}$ the grade of $T^{A}$ in the Lie superalgebra, and note that the structure constants are antisupersymmetric in the first two indices.

$$
f_{C}^{A B}=-(-1)^{\eta_{A} \eta_{B}} f^{B A}{ }_{C}
$$

Furthermore, using the invariance of the supertrace

$$
\operatorname{Str}\left(T^{A}\left[T^{B}, T^{C}\right]\right)=\operatorname{Str}\left(\left[T^{A}, T^{B}\right] T^{C}\right)
$$

we find that the structure constants are also antisupersymmetric in the first and last indices.

$$
f_{C}^{A B}=-(-1)^{\eta_{A} \eta_{C}} f_{A}^{C B}
$$

The $x_{A}$ commute (resp. anticommute) whenever the $T^{A}$ are graded even (resp. odd), and so we can define without ambiguity $\eta_{A}$ to be the gradation of $x_{A}$ in the Grassmann algebra. The local currents from the PCM are constructed from elements of this Lie algebra.

We are interested in the Lie superalgebras $s u(m \mid n)$, and $o s p(m \mid 2 n)$, satisfying

$$
\begin{aligned}
s u(m \mid n) & : \quad \operatorname{Str}(X)=0, X=-X^{\dagger} \\
o s p(m \mid 2 n) & : \quad X^{S T}=-H X H^{-1}
\end{aligned}
$$

For the Lie superalgebras $s u(m \mid n)(m \neq n)$ and $\operatorname{sep}(m \mid 2 n)$ we can define a non-degenerate invariant bilinear form $G^{A B}=\operatorname{Str}\left(T^{A} T^{B}\right)$. Note that this implies that $G^{A B}=0$ unless $\eta_{A}=\eta_{B}$ (consistency) and that $G^{A B}=$ $(-1)^{\eta_{A}} G^{B A}$ (supersymmetry). We define the inverse $G_{A B}$ via

$$
G_{A B} G^{B C}=\delta_{A}^{C}
$$

Note in particular that this implies that

$$
G^{A B} X_{A} Y_{B}=X_{A} Y^{A}=(-1)^{\eta_{A}} X^{A} Y_{A}=G_{B A} X^{A} Y^{B}
$$


There is a one-dimensional ideal of $s u(n \mid n)$, generated by $i \mathbb{I}_{2 n}$. It is therefore not semi-simple and has no non-degenerate metric, and it is usual to consider the PCM on $P S U(n \mid n)=S U(n \mid n) / \mathbb{I}_{2 n}$. The Killing form for this is exactly zero (it is proportional to the dual Coxeter number) but an invariant bilinear form does exist. We shall avoid this by later imposing $m \neq n$.

We will make much use of the completeness condition in what is to follow. For any $X=X_{A} T^{A} \in \mathrm{g}$ we have

$$
X_{A}=\operatorname{Str}\left(T_{A} X\right)
$$

\section{Local Charges of the PCM}

The PCM is defined by the lagrangian

$$
\mathcal{L}=\frac{\kappa}{2} \operatorname{Str}\left(\partial_{\mu} g^{-1} \partial^{\mu} g\right)
$$

where $g$ takes values in a supergroup $G$, either $S U(m \mid n)$ or $O S p(m \mid 2 n) . \kappa$ is a dimensionless constant, its value unimportant for the classical model, and $\mu$ indexes the $1+1 \mathrm{D}$ spacetime. This lagrangian is invariant under a global chiral symmetry $g(x, t) \rightarrow g_{1} g(x, t) g_{2}^{-1}$, with associated Noether conserved local currents

$$
j_{\mu}^{L}=\kappa \partial_{\mu} g g^{-1} \quad, \quad j_{\mu}^{R}=-\kappa g^{-1} \partial_{\mu} g .
$$

The conservation of these currents are the equations of motion. These currents belong to the even subspace of $\mathbf{g} \otimes \Lambda$.

From these conserved currents it is possible to construct the higher spin local and non-local conserved charges. Using either the left or right currents will give rise to identical local charges. The non-local charges constructed from them are not equal, but form two copies of a super-Yangian structure. When there is no confusion, we shall drop the $L / R$ indices.

We assume appropriate boundary conditions on $j_{\mu}(x)$

$$
j_{\mu}(x)=0 \quad \text { as } x \rightarrow \pm \infty .
$$

The currents are conserved, and satisfy the Bianchi identity

$$
\partial^{\mu} j_{\mu}=0 \quad, \quad \partial_{\mu} j_{\nu}-\partial_{\nu} j_{\mu}-\frac{1}{\kappa}\left[j_{\mu}, j_{\nu}\right]=0
$$

Immediately we can form conserved charges out of the Noether currents (21) by $Q^{(0)}=\int d x j_{0}$. More importantly though, conditions (23) allow us to

form a local conserved charge of spin $s$ through the use of a $G$-invariant tensor of degree $s+1$. Although there are infinitely many such invariant 
tensors, there are only finitely many independent (primitive) tensors. The number is equal to the rank of $\mathbf{g}$. All other invariants are constructed from these primitive ones.

The intention is to find a maximal set of mutually commuting conserved local charges $\left\{q_{s}\right\}$. The existence of these currents displays the integrable nature of the PCM, because (equivalent to the construction of non-local charges) the two conditions in (23) allow a Lax pair to be constructed.

It will prove useful to write conditions (23) in terms of light-cone coordinates, $x^{ \pm}=\frac{1}{2}(t \pm x)$

$$
\partial_{-} j_{+}=-\partial_{+} j_{-}=-\frac{1}{2 \kappa}\left[j_{+}, j_{-}\right]
$$

\subsection{The Energy-Momentum Tensor and Conformal Invari- ance}

The energy-momentum tensor is the variation of the lagrangian with respect to the spacetime metric,

$$
T_{\mu \nu}=-\frac{1}{2 \kappa}\left(\operatorname{Str}\left(j_{\mu} j_{\nu}\right)-\frac{1}{2} \eta_{\mu \nu} \operatorname{Str}\left(j_{\rho} j^{\rho}\right)\right)
$$

This is traceless, symmetric and conserved. In light-cone coordinates

$$
T_{ \pm \pm}=-\frac{1}{2 \kappa} \operatorname{Str}\left(j_{ \pm} j_{ \pm}\right), \quad T_{+-}=T_{-+}=0
$$

and

$$
\partial_{-} T_{++}=\partial_{+} T_{--}=0
$$

Here $T_{+-}$is the trace of the two-dimensional energy-momentum and its vanishing implies that the model has classical conformal invariance. The situation is more complicated in the presence of quantum anomalies. The one loop beta function is proportional to $h^{\vee}$, the dual Coxeter number of the Lie (super)algebra [12]. For purely bosonic Lie algebras, $h^{\vee} \neq 0$, and so a WZ term must be added to the lagrangian for conformal invariance in the quantum model [9]. However there are some Lie superalgebras (namely $\operatorname{psl}(n \mid n)$ and $\operatorname{sep}(2 n+2 \mid 2 n))$ for which $h^{\vee}=0$, and these models retain conformal invariance in the quantum model, at least to one loop [8, 13].

We note here that we can form a series of higher-spin conservation laws,

$$
\partial_{-}\left(T_{++}^{p}\right)=\partial_{+}\left(T_{--}^{p}\right)=0 .
$$

These give the classical conformal symmetry of the model, but are not expected to be preserved for the quantum models for which the dual Coxeter number is non-zero. We shall not be concerned with these directly however, as we shall see that more general higher-spin currents can be formed. 


\subsection{Canonical Formalism}

Our aim here is to calculate the super-Poisson brackets (SPBs) for the current components $j_{\mu A}$ [22]. As in the bosonic case [9, 23, it is convenient to introduce the non-local operator $\Delta_{1}=\partial_{1}-\frac{1}{\kappa}\left[j_{1},\right]$ using which the Bianchi identity is re-expressed as $j_{0}=\Delta_{1}^{-1}\left(\partial_{0} j_{1}\right)$. We can now write the action as a functional of $j_{1}(x)$ only

$$
\mathcal{L}=\frac{1}{2 \kappa} \operatorname{Str}\left(\left(\Delta_{1}^{-2} \partial_{0} j_{1}\right)\left(\partial_{0} j_{1}\right)-j_{1}^{2}\right)
$$

(where we have imposed suitable boundary conditions such that $\Delta_{1}^{-1}(A) B=$ $-A \Delta_{1}^{-1}(B)$ up to a total divergence).

Defining the conjugate momentum of $j_{1}$ to be $\pi=\pi^{A} T_{A}$, where $\pi^{A}=$ $\partial \mathcal{L} / \partial\left(\partial_{0} j_{1 A}\right)$ we find that $j_{0}=-2 \kappa \Delta_{1} \pi$. Then, using $\left\{j_{1 A}(x), \pi^{B}(y)\right\}=$ $\delta_{A}^{B} \delta(x-y)$, we find that

$$
\begin{aligned}
\left\{j_{0 A}(x), j_{0 B}(y)\right\}= & (-1)^{\chi} f_{A B}{ }^{C} j_{0 C}(x) \delta(x-y) \\
\left\{j_{0 A}(x), j_{1 B}(y)\right\}= & (-1)^{\chi} f_{A B}{ }^{C} j_{1 C}(x) \delta(x-y) \\
& +\kappa G_{A B} \partial_{x} \delta(x-y) \\
\left\{j_{1 A}(x), j_{1 B}(y)\right\}= & 0
\end{aligned}
$$

where $\chi=\eta_{A} \cdot \eta_{B}+\eta_{A}+\eta_{B}$. In light-cone coordinates these become

$$
\begin{aligned}
\left\{j_{ \pm A}(x), j_{ \pm B}(y)\right\}= & (-1)^{\chi} f_{A B} C\left(\frac{3}{2} j_{ \pm C}(x)-\frac{1}{2} j_{\mp C}(x)\right) \delta(x-y) \\
& \pm 2 \kappa G_{A B} \delta^{\prime}(x-y) \\
\left\{j_{+A}(x), j_{-B}(y)\right\}= & \frac{1}{2}(-1)^{\chi} f_{A B}^{C}\left[j_{+C}(x)+j_{-C}(y)\right] \delta(x-y)
\end{aligned}
$$

These are very similar in form to the Poisson brackets of the bosonic model [9] but we must take account of the non-trivial gradings.

\subsection{Higher-Spin Conserved Charges}

We can construct the Noether $G_{L} \times G_{R}$ conserved charges

$$
Q_{A}^{0}=\int_{-\infty}^{\infty} d x j_{0 A}(x)
$$

for both left and right currents $j_{\mu}^{L}$ and $j_{\mu}^{R}$. More interestingly we can use the above SPBs to find an infinite set of commuting higher-spin holomorphic 
local currents. We shall obtain the same set of local currents if we use either the left or right current. We first note that, similar to the bosonic model 9], to every invariant tensor $d^{A_{1} \ldots A_{p}}$ (supersymmetric in adjacent indices) associated with a Casimir element of degree $p$, we can associate a conserved current of spin $p$. Denote such a Casimir element

$$
\mathcal{C}^{p}=d^{A_{1}, \ldots A_{p}} T_{A_{1}} \ldots T_{A_{p}}
$$

and supersymmetry means that

$$
d^{A_{1} \ldots A_{k} A_{k+1} \ldots A_{p}}=(-1)^{\eta_{A_{k}} \eta_{A_{k+1}}} d^{A_{1} \ldots A_{k+1} A_{k} \ldots A_{p}}
$$

We note that, for $s u(m \mid n)$ and $\operatorname{sep}(m \mid 2 n), d^{A_{1}, \ldots A_{p}}=0$ unless $\sum \eta_{A_{i}}=0$. Indeed, Casimir elements are bosonic for all basic superalgebras except $Q(n)$ [19. Invariance then implies that

$$
\left[\mathcal{C}^{p}, T^{B}\right]=0 \Rightarrow \sum_{i=1}^{p}(-1)^{\eta_{B}\left(\eta_{A_{i}}+\ldots \eta_{A_{p}}\right)} d^{A_{1} \ldots \hat{A}_{i} C \ldots A_{p}} f_{C}^{A_{i} B}=0
$$

We define the action of this tensor on an element of the Lie superalgebra $X=X_{A} T^{A}$ by

$$
d^{(p)}(X)=d^{A_{1} \ldots A_{p}} X_{A_{1}} \ldots X_{A_{p}}
$$

and observe that it is $G$-invariant

$$
d^{(p)}\left(g X g^{-1}\right)=d^{(p)}(X) \text { for } g \in G .
$$

We can simplify matters by noting that every element of a Lie (super)algebra is locally conjugate to some element of its Cartan subalgebra (CSA), $\mathbf{h} \subset \mathbf{g}$ i.e. its maximal abelian subgroup. For most basic Lie superalgebras, $\mathbf{h}$ is the CSA of its bosonic subalgebra $\mathbf{h} \subset \mathbf{g}_{\overline{0}}$.

Using the invariance property we then have

$$
d^{(p)}(X)=d^{(p)}(H)=d^{a_{1} \ldots a_{p}} H_{a_{1}} \ldots H_{a_{p}}
$$

where $H=H_{a} T^{a}=g X g^{-1} \in \mathbf{h}$. We can thus consider the invariant tensor to be restricted to the CSA, and so we are only interested in the invariants of the underlying bosonic Lie subalgebra. ${ }^{1}$

To each superalgebra there are an infinite number of invariant tensors, notably of the form $\operatorname{Str}\left(T^{A_{1}} \ldots T^{A_{m}}\right)$, but there are only finite many independent (or primitive) tensors, the number being equal to the rank of the superalgebra. All local conserved charges will be generated by charges formed from these primitive tensors. These primitive tensors were discussed in 24, 25] for the bosonic algebras, and for superalgebras in [26, 27.

\footnotetext{
${ }^{1}$ The exception is the strange superalgebra $Q(n)$, where $\mathbf{h} \cap \mathbf{g}_{\overline{1}} \neq \emptyset[19$.
} 
An infinite set of higher spin conserved charges can be constructed from tensors which satisfy (35). Using (24) it is straightforward to verify that

$$
\partial_{-}\left(d^{A_{1} \ldots A_{p}} j_{+A_{p}} \ldots j_{+A_{1}}\right)=0
$$

where the change in ordering of the indices is non-trivial because of the $\mathbb{Z}_{2}$-grading (34). Then the local conserved charges are

$$
q_{ \pm s}=\int d x d^{A_{1} \ldots A_{s+1}} j_{ \pm A_{s+1}}(x) \ldots j_{ \pm A_{1}}(x) .
$$

The charges are labelled by $s$, their spin. The Poisson bracket with the (purely bosonic) boost generator $M$ is $\left\{M, q_{ \pm s}\right\}= \pm s q_{ \pm s}$. We shall define the charges $q_{ \pm s}$ with $s>0$ as having positive/negative chirality. The additive nature of these local charges implies that they have a trivial coproduct

$$
\Delta\left(q_{ \pm s}\right)=q_{ \pm s} \otimes \mathbb{I}+\mathbb{I} \otimes q_{ \pm s}
$$

Using the invariance condition (35), it is simple to show that $\left\{q_{+r}, q_{-s}\right\}=0$ for any integers $r, s>0$. Furthermore, for charges of equal chirality, only the non-ultra local terms contribute.

$$
\begin{aligned}
\left\{q_{ \pm r}, q_{ \pm s}\right\}= & \pm 2(r+1)(s+1) \kappa \int d x(-1)^{\eta_{B}\left(\eta_{B_{1}}+\ldots+\eta_{B_{s}}\right)} d^{A_{1} \ldots A_{r} A} d^{B_{1} \ldots B_{s} B} G_{A B} \\
& \times j_{ \pm A_{1}} \ldots j_{ \pm A_{r}} \partial_{x}\left(j_{ \pm B_{1}} \ldots j_{ \pm B_{s}}\right)
\end{aligned}
$$

We are interested in the currents formed from a tensor with components $d^{A_{1} \ldots A_{r}}=\operatorname{sStr}\left(T^{A_{1}} \ldots T^{A_{r}}\right)$, which satisfies (35). sStr denotes the normalised supersymmetric supertrace

$$
\operatorname{sStr}\left(T^{A_{1}} T^{A_{2}} \ldots T^{A_{r}}\right)=\frac{1}{r !} \sum_{\sigma \in S_{r}} \epsilon_{\sigma} \operatorname{Str}\left(T^{A_{\sigma(1)}} T^{A_{\sigma(2)}} \ldots T^{A_{\sigma(r)}}\right)
$$

where $S_{r}$ is the symmetric group of degree $r$, and $\epsilon_{\sigma}=-1$ if $\sigma$ involves an odd number of permutations of fermionic indices, and equals 1 otherwise. This tensor is exactly zero unless $\eta_{A_{1}}+\ldots+\eta_{A_{r}}=0 \bmod 2$. This gives rise to holomorphic currents $\mathcal{J}_{ \pm r}=\operatorname{Str}\left(j_{ \pm}^{r}\right)$, and associated conserved charges

$$
q_{ \pm(r-1)}=\int d x \operatorname{Str}\left(j_{ \pm}^{r}\right)
$$

and we find that that equation (42) simplifies to

$$
\left\{q_{ \pm r}, q_{ \pm s}\right\}= \pm 2(r+1)(s+1) \kappa \int d x \operatorname{Str}\left(j_{ \pm}^{r} T_{A}\right) \partial_{x} \operatorname{Str}\left(j_{ \pm}^{s} T^{A}\right) .
$$

We now want to find the currents $\mathcal{J}_{r}(x)$ (or indeed, algebraic functions of them) which give rise to charges in involution. In particular we note that $q_{ \pm 2}$ always commutes with the other charges, showing that all higher-spin charges are classically in involution with energy-momentum.

We must now deal separately with the cases $G=S U(m \mid n)$ and $G=$ $O S p(m \mid 2 n)$, and we shall see that in the former case we must impose $m \neq n$. 


\subsection{Commuting Charges for $S U(m \mid n)$}

It is evident that $X \in s u(m \mid n)$ does not imply $X^{p} \in s u(m \mid n)$ for all integers $p$ because supertracelessness will not in general hold. In the case $m \neq n$ we can replace $j_{+}^{r}$ by the supertraceless and anti-hermitian $j_{+}^{r}-$ $(1 / l) \operatorname{Str}\left(j_{+}^{r}\right) \mathbb{I}_{m+n}$ where $l=m-n$, and then using the completeness condition (19) we find

$$
\left\{q_{ \pm r}, q_{ \pm s}\right\}=\mp \frac{2(r+1)(s+1) \kappa}{l} \int d x \operatorname{Str}\left(j_{ \pm}^{r}\right) \partial_{x} \operatorname{Str}\left(j_{ \pm}^{s}\right)
$$

This term is not in general zero. It is necessary to know the exact form of the Poisson brackets for $\mathcal{J}_{ \pm r}=\operatorname{Str}\left(j_{ \pm}^{r}\right)$. After some computation the result is

$$
\begin{aligned}
\left\{\mathcal{J}_{r}(x), \mathcal{J}_{s}(y)\right\} & =\left(\frac{r s}{l} \mathcal{J}_{r-1}(x) \mathcal{J}_{s-1}(x)-r s \mathcal{J}_{r+s-2}(x)\right) \delta^{\prime}(x-y) \\
& +\left(\frac{r s}{l} \mathcal{J}_{r-1}(x) \mathcal{J}_{s-1}^{\prime}(x)-\frac{r s(s-1)}{(r+s-2)} \mathcal{J}_{r+s-2}^{\prime}(x)\right) \delta(x-y)
\end{aligned}
$$

Note the similarity between this and the Poisson brackets for the currents of the bosonic $S U(l)$ model [9]. Indeed, these Poisson brackets are all antisymmetric, and feature purely bosonic currents. This is also the point where we must distinguish between the quantum conformal model $P S U(n \mid n)$ and the non-conformal models.

We now want to find a set of algebraically independent currents which give rise to a set of mutually commuting charges. We follow a similar method to [9] and define a generating function $A(x, \lambda)$ with a spectral parameter $\lambda$ by

$$
A(x, \lambda)=\operatorname{sdet}\left(1-\lambda j_{+}(x)\right)=\exp \left(-\sum_{r=2}^{\infty} \frac{\lambda^{r}}{r} \mathcal{J}_{r}(x)\right)
$$

and then claim that the set of currents defined by

$$
\mathcal{K}_{r+1}(x)=\left.A(x, \lambda)^{r / l}\right|_{\lambda^{r+1}}
$$

form commuting charges upon integration over space.

$$
\left.\int d x d y\left\{A(x, \mu)^{r / l}, A(y, \nu)^{s / l}\right\}\right|_{\mu^{r+1} \nu^{s+1}}=0
$$

This differs from the bosonic model [9] in that (48) is not a polynomial of finite order in $\lambda$ (as it is in the bosonic case), but a rational function. For the bosonic model, when $r \equiv 0 \bmod l$ current (49) would be exactly zero. The local charges therefore have spins equal to the exponents of the Lie algebra modulo its Coxeter number $h$. No such pattern seems to exist for 
the supergroup model, and (49) seems to be non-zero for all positive integer values of $r$.

However after noting this, we can proceed with an analogous argument. We calculate $\{\ln A(x, \mu), \ln A(y, \nu)\}$ using (47), and thence it is seen that (50) is satisfied.

The infinite number of currents (49) are not algebraically independent. The number of independent currents is equal to the rank of the superalgebra, so for $s u(m \mid n)$ there are $m+n-1$ independent currents. Therefore a maximal set of algebraically independent currents which form commuting charges are

$$
\left\{K_{i}(x) \mid 2 \leq i \leq m+n\right\} .
$$

Any higher spin currents which give further commuting charges must necessarily be algebraic functions of these currents. The form of the currents is identical to that of $S U(m-n)$, , but we shall reproduce the first few examples of them here for completeness.

$$
\begin{aligned}
\mathcal{K}_{2} & =\mathcal{J}_{2} \\
\mathcal{K}_{3} & =\mathcal{J}_{3} \\
\mathcal{K}_{4} & =\mathcal{J}_{4}-\frac{3}{2(m-n)} \mathcal{J}_{2}^{2} \\
\mathcal{K}_{5} & =\mathcal{J}_{5}-\frac{10}{3(m-n)} \mathcal{J}_{3} \mathcal{J}_{2}
\end{aligned}
$$

\subsection{Commuting Charges for $O S p(m \mid 2 n)$}

Using the defining relation for this superalgebra, we see that $X \in \operatorname{osp}(m \mid 2 n)$ implies that $X^{p} \in o s p(m \mid 2 n)$ if $p$ is odd. So for such $p$, we can use the completeness condition (19) to show that the integrand of (45) is a total divergence, and thus that the charges commute.

$$
\left\{q_{ \pm r}, q_{ \pm s}\right\}= \pm \frac{2 s(r+1)(s+1) \kappa}{r+s} \int d x \partial_{x} \operatorname{Str}\left(j_{ \pm}^{r+s}\right)=0
$$

So for the $O S p(m \mid 2 n)$ model, the set of $q_{r}$ defined by (44) for $r$ odd is a set of mutually commuting charges. (For $r$ even, these charges are exactly zero.)

We will find that we can derive more interesting conserved charges if we calculate the equal-time Poisson brackets for the currents using (31).

$$
\left\{\mathcal{J}_{r}(x), \mathcal{J}_{s}(y)\right\}=-r s \mathcal{J}_{r+s-2}(x) \delta^{\prime}(x-y)-\frac{r s(s-1)}{(r+s-2)} \mathcal{J}_{r+s-2}^{\prime}(x) \delta(x-y)
$$


This equation holds for all $r, s \geq 1$, but is only interesting for even $r, s$. Again we note the similarity between these relations and those given for the bosonic orthogonal and symplectic algebras [9]. Analogous to these models we will find a family of commuting currents, with a free parameter $\alpha$. We can formulate these currents through the use of generating functions with a parameter $\lambda$. We define

$$
B(x, \lambda)=\operatorname{sdet}\left(1-\sqrt{\lambda} j_{+}(x)\right)=\exp \left(-\sum_{r=1}^{\infty} \frac{\lambda^{r}}{2 r} \mathcal{J}_{2 r}(x)\right)
$$

and find that the currents defined by

$$
\mathcal{K}_{r+1}(x)=\left.B(x, \lambda)^{\alpha r}\right|_{\lambda^{(r+1) / 2}}
$$

give rise to a mutually commuting set of currents

$$
\left.\int d x d y\left\{B(x, \mu)^{\alpha r}, B(y, \nu)^{\alpha s}\right\}\right|_{\mu^{(r+1) / 2} \nu^{(s+1) / 2}}=0 .
$$

The argument proceeds similarly to the unitary case. The difference now is that we have a family of commuting charges, depending on a free parameter $\alpha$. The resulting currents are similar in form to the orthogonal and symplectic cases [9]. We reproduce them here.

$$
\begin{aligned}
\mathcal{K}_{2} & =\mathcal{J}_{2} \\
\mathcal{K}_{4} & =\mathcal{J}_{4}-\frac{3 \alpha}{2} \mathcal{J}_{2}^{2} \\
\mathcal{K}_{6} & =\mathcal{J}_{6}-\frac{15 \alpha}{4} \mathcal{J}_{4} \mathcal{J}_{2}+\frac{25 \alpha^{2}}{8} \mathcal{J}_{2}^{3} \\
\mathcal{K}_{8} & =\mathcal{J}_{8}-\frac{14 \alpha}{3} \mathcal{J}_{6} \mathcal{J}_{2}-\frac{7 \alpha}{4} \mathcal{J}_{4}^{2}+\frac{49 \alpha^{2}}{4} \mathcal{J}_{4} \mathcal{J}_{2}^{2}-\frac{343 \alpha^{3}}{48} \mathcal{J}_{2}^{4}
\end{aligned}
$$

\subsubsection{The Superpfaffian}

For $S O(2 l)$ there is another conserved current which cannot be expressed as the trace of a power of $j_{+}(x)$ : the Pfaffian current of spin $l$,

$$
\mathcal{P}(x)=\epsilon^{I_{1} J_{1} \ldots I_{l} J_{l}}\left(j_{+}\right)_{I_{1} J_{1}} \ldots\left(j_{+}\right)_{I_{l} J_{l}}
$$

By requiring that the charge associated to this current is in involution with the set of commuting charges formed from traces, the value of $\alpha$ is fixed to be $1 /(2 l-2)$ [9. A similar situation exists for $O S p(2 m \mid 2 n)$, for which there exists an analogous current, the superpfaffian current of spin $m-n$ 14. Its charge is in involution with the supertrace currents fixes $\alpha$ to be $1 /(2 m-2 n-2)$. The superpfaffian current differs from the others already considered in that it cannot be written in the form $d^{A_{1} \ldots A_{p}} j_{ \pm A_{1}} \ldots j_{ \pm}$, but is instead a rational function. 
Given any supermatrix written in block form $\left(\begin{array}{cc}A & B \\ C & D\end{array}\right)$ for which $D$ is invertible, we define the superpfaffian to be

$$
\operatorname{Spf}\left(\begin{array}{cc}
A & B \\
C & D
\end{array}\right)=\frac{\operatorname{Pfaff}\left(A-B D^{-1} C\right)}{\sqrt{\operatorname{det}(\mathrm{D})}}=\frac{\operatorname{Pfaff}(A)}{\sqrt{\operatorname{det}\left(D-C A^{-1} B\right)}}
$$

where Pfaff is the ordinary pfaffian.

Let $j_{+}(x) \in \operatorname{osp}(2 m \mid 2 n)$. Every element of a Lie superalgebra is locally conjugate to some element of the Cartan subalgebra. So there exists $U(x) \in$ $O S p(2 m \mid 2 n)$ such that

$U j_{+} U^{-1}=\operatorname{diag}\left(\left[\begin{array}{cc}0 & \lambda_{1} \\ -\lambda_{1} & 0\end{array}\right], \ldots,\left[\begin{array}{cc}0 & \lambda_{m} \\ -\lambda_{m} & 0\end{array}\right], i \mu_{1}, \ldots i \mu_{n},-i \mu_{1}, \ldots,-i \mu_{n}\right)$

for real $\lambda_{i}(x), \mu_{j}(x)$ (the weights) [10. The currents that we are interested in can be expressed as functions of the $m+n$ weights.

We first note that we can write (55) as

$$
\operatorname{sdet}\left(1-\sqrt{\nu} j_{+}(x)\right)=\frac{\left(1+\nu \lambda_{1}^{2}\right) \ldots\left(1+\nu \lambda_{m}^{2}\right)}{\left(1+\nu \mu_{1}^{2}\right) \ldots\left(1+\nu \mu_{n}^{2}\right)}
$$

and then the supertrace currents (56) which give commuting charges can be expressed in terms of the weights as

$$
\mathcal{K}_{p}(x)=\left.\left[\frac{\left(1+\nu \lambda_{1}^{2}\right) \ldots\left(1+\nu \lambda_{m}^{2}\right)}{\left(1+\nu \mu_{1}^{2}\right) \ldots\left(1+\nu \mu_{n}^{2}\right)}\right]^{\alpha(p-1)}\right|_{\nu^{p / 2}}
$$

or equivalently as

$$
\mathcal{K}_{p}(x)=\left.\exp \left(-\alpha(p-1) \sum_{r=1} \frac{(-1)^{r} \nu^{r}}{r}\left(\sum_{i} \lambda_{i}^{2 r}-\sum_{j} \mu_{j}^{2 r}\right)\right)\right|_{\nu^{p / 2}}
$$

Our conserved superpfaffian current can then be written in terms of the weights as

$$
\mathcal{P}(x)=\operatorname{spf}\left(j_{+}(x)\right)=\left|\frac{\lambda_{1} \ldots \lambda_{m}}{\mu_{1} \ldots \mu_{n}}\right|
$$

and our claim is that requiring $\int d x d y\left\{\mathcal{K}_{p}(x), \mathcal{P}(y)\right\}=0$ for all (even) $p$ will constrain $\alpha$ to be $(2 m-2 n-2)^{-1}$. We shall use the Poisson bracket relations

$$
\begin{aligned}
& \left\{\lambda_{i}(x), \lambda_{j}(y)\right\}=-4 \kappa \delta_{i j} \delta^{\prime}(x-y) \\
& \left\{\mu_{k}(x), \mu_{l}(y)\right\}=4 \kappa \delta_{k l} \delta^{\prime}(x-y)
\end{aligned}
$$


(where the difference in signs comes from the definition of supertrace) to calculate (writing $C(x)=\operatorname{sdet}\left(1-\sqrt{\nu} j_{+}(x)\right)$ and $\beta=\alpha(p-1)$ for convenience)

$$
\begin{gathered}
\int d x d y\left\{\mathcal{P}(x), C(y)^{\beta}\right\}=\int d x d y \beta C(y)^{\beta-1}\{\mathcal{P}(x), C(y)\} \\
=8 \beta \kappa \nu \int d x\left(\sum_{i=1}^{m} \partial_{x}\left(\frac{\mathcal{P}(x)}{\lambda_{i}(x)}\right) \frac{C(x)^{\beta} \lambda_{i}(x)}{1+\nu \lambda_{i}^{2}(x)}-\sum_{k=1}^{n} \partial_{x}\left(\frac{\mathcal{P}(x)}{\mu_{k}(x)}\right) \frac{C(x)^{\beta} \mu_{k}(x)}{1+\nu \mu_{k}^{2}(x)}\right) \\
=\quad \\
8 \beta \kappa \nu \int d x\left[(m-n)-\frac{\nu}{\beta} \partial_{\nu}-1-\frac{1}{2 \beta}\right]\left(\partial_{x} \mathcal{P}(x)\right) C(x)^{\beta}
\end{gathered}
$$

We are only interested in the coefficient of $\nu^{p / 2}$, and so we replace $\nu \partial_{\nu} \mapsto$ $p / 2-1$. We then find that the term in square brackets vanishes if and only if $\alpha=1 /(2 m-2 n-2)$, as required.

We immediately see that there is a similar pattern of spins for the local charges on $O S p(m \mid 2 n)$ and $S O(m-2 n)$; Saleur and Kaufmann [12 have studied the similarity between the S-matrices of the models with these symmetries.

Interestingly, for the $O S p(2 n+2 \mid 2 n)$ models (precisely those which are exactly conformal) there exists no finite value of $\alpha$ for which the superpfaffian charge commutes with all the other charges. This does not affect their integrability properties as it is still possible to construct a Lax pair.

\section{Non-Local Charges}

In addition to the local charges, there are two infinite sets of conserved nonlocal charges, which are elements of $(\mathbf{g} \otimes \Lambda)_{\overline{0}}$ and generate a chiral Yangian structure $Y(\mathbf{g})_{L} \times Y(\mathbf{g})_{R}$ [9, 28].

The full set of non-local charges are generated by the conserved local charge

$$
Q_{A}^{(0)}=\int_{-\infty}^{\infty} d x j_{0 A}(x)
$$

and the first non-local charge

$$
Q_{A}^{(1)}=\int_{-\infty}^{\infty} d x j_{1 A}(x)-\frac{1}{2 \kappa} f_{A}^{B C} \int_{-\infty}^{\infty} d x j_{0 B}(x) \int_{-\infty}^{x} d y j_{0 C}(y)
$$

where conservation follows from (23). Higher charges are formed from commutations of these. Their construction in [28, 29] for the bosonic charges can be applied analogously here. 
To show that all local charges are in involution with the non-local charges, it suffices to show that the local charges commute with the first two charges $Q_{A}^{(0)}$ and $Q_{A}^{(1)}$.

The invariance of the d-tensor can be used in a straightforward fashion to show that

$$
\left\{q_{s}, Q_{A}^{(0)}\right\}=0
$$

Commutation of $Q_{A}^{(1)}$ is not so simple to show, but we can proceed by using a similar argument to 9]. We consider each of the two terms of $Q_{B}^{(1)}$ separately, and using the invariance property (35), we find that the commutation of the first term gives

$$
\left\{q_{s}, \int d y j_{1 B}(y)\right\}=-(s+1) \int d x d^{C A_{1} \ldots A_{s}} f_{C}{ }_{B} j_{+A_{s}} \ldots j_{+A_{1}} j_{1 D}
$$

As in [9], when looking at the second term we must be cautious when working with the limits of the spatial integration. We thus integrate between $\pm L$ and then take the limit $\mathrm{E} \rightarrow \infty$. We are interested in

$$
\left\{q_{s}, \int_{-L}^{L} d y \int_{-L}^{y} d z f_{B}^{C D} j_{0 C}(y) j_{0 D}(z)\right\}
$$

To evaluate this, we then introduce a step function,

$$
\int_{-L}^{L} d x \int_{-L}^{L} d y \int_{-L}^{L} d z d^{A_{1} \ldots A_{s+1}} f_{B}^{C D}\left\{j_{+A_{s+1}}(x) \ldots j_{A_{1}}(x), j_{0 C}(y) j_{0 D}(z)\right\} \theta(y-z)
$$

All the ultra-local terms (i.e. the $\delta(x-y)$ and $\delta(x-z)$ ) vanish by invariance (35), and after some computation (noting that the currents vanish at infinity) we are left with

$$
2 \kappa(s+1) \int d x d^{C A_{1} \ldots A_{s}} f_{C}{ }^{D}{ }_{B} j_{+A_{s}} \ldots j_{+A_{1}} j_{0 D}
$$

We recombine results (71) and (74), and again use the invariance property (35) to show the final result

$$
\left\{q_{s}, Q_{A}^{(1)}\right\}=0
$$

The Yangian charges do not in general commute.

$$
\begin{aligned}
& \left\{Q_{A}^{(0)}, Q_{B}^{(0)}\right\}=(-1)^{\chi} f_{A B}{ }^{C} Q_{C}^{(0)} \\
& \left\{Q_{A}^{(0)}, Q_{B}^{(1)}\right\}=(-1)^{\chi} f_{A B}{ }^{C} Q_{C}^{(1)}
\end{aligned}
$$

where $\chi=\eta_{A} \cdot \eta_{B}+\eta_{A}+\eta_{B}$. Once equipped with the additional structure of a (non-trivial) coproduct and counit, this is the expected form of the super-Yangian algebra [16, 17, 18, 


\section{Remarks on the Quantum Model}

To determine whether the higher spin local currents are also conserved in the quantum theory, we use the anomaly counting method of Goldschmidt and Witten [15. This is a rather indirect method, and does not convey an insight into the form of these anomalies. Instead it tries to show that any quantum anomalies can be written in the form of a total derivative, in which case a conservation law still exists, although in a modified form.

We must consider all possible anomaly terms which have the same behaviour under the symmetries of the model. These symmetries comprise of the continuous Lorentz and chiral symmetries, and also some discrete symmetries.

For any supergroup, the principal chiral model is invariant under the map $\pi$ : $g \mapsto g^{-1}$. This exchanges the left and right currents. Additional symmetries arise as outer autmorphisms of the underlying Lie superalgebras [19.

$$
\begin{gathered}
\gamma: g \mapsto g^{*} \quad \text { for } \mathbf{g}=s u(m \mid n) \\
\sigma: g \mapsto M G M^{-1} \quad \text { for } \mathbf{g}=\operatorname{osp}(2 m \mid 2 n)
\end{gathered}
$$

where $M$ is an element of $O S P(2 m \mid 2 n)$ with superdeterminant - 1 . Each of the currents $\mathcal{J}_{r}(x)$ are either odd or even under the action of all of these symmetries. The argument proceeds identically to the bosonic PCM 9, and we shall not reproduce it all here, but instead illustrate the idea with the spin 2 example. It does not matter for the following whether we are considering $s u(m \mid n)$ or $\operatorname{ssp}(m \mid 2 n)$; the results are the same for all models.

There are only two spin 2 currents, $\mathcal{J}_{2}=\operatorname{Str}\left(j_{ \pm}^{2}\right)$. (We shall just consider the + current, the argument is identical for the other.) This is even under the discrete symmetries, invariant under the chiral symmetry, and of mass dimension 2. There is only one possible anomaly term with identical behaviour, $\operatorname{Str}\left(j_{-} \partial_{+} j_{+}\right)$, which can be written as a total derivative $\partial_{+} \operatorname{Str}\left(j_{-} j_{+}\right)$. So the only possible correction to the conservation law is

$$
\partial_{-} \mathcal{J}_{2}=\alpha \partial_{+} \operatorname{Str}\left(j_{-} j_{+}\right)
$$

where $\alpha$ is some unknown parameter, and we have a modified conservation law. (Note that $\alpha$ can be zero, and we would expect it to be so for those models which retain quantum conformal invariance.)

Similar results hold for spin 3 and 4 currents, but not for higher spins. This does not mean that there is no quantum conserved current, for the anomaly counting method is sufficient but not necessary. Nevertheless, integrability is guaranteed by the existence of one higher spin conservation law [30]. 


\section{Conclusions and Further Questions}

We have derived a set of commuting charges for the principal chiral model on the Lie supergroups $S U(m \mid n)$ for $m \neq n$, and $O S p(m \mid 2 n)$. These are integrals of local currents, each constructed with the use of an invariant of the underlying Lie superalgebra.

The $S U(m \mid n)$ models have conserved currents generated by $m+n-1$ primitive currents. The current algebra is similar in appearance to that of $S U(m-n)$.

The orthosymplectic models $O S p(2 m \mid 2 n)$ and $O S p(2 m+1 \mid 2 n)$ have each $m+n$ primitive currents from which the infinite charges can be calculated. For the former set of models, the superpfaffian charge will only commute with the other charges if $m \neq n+1$. The algebra of currents suggests a relationship between $O S p(m \mid 2 n)$ and $S O(m-2 n)$ models [12.

In the bosonic model there is a correlation between the degrees of the primitive currents and the exponents of the underlying Lie algebra 9 . This same pattern of currents exists for the affine Toda field theories [31, 32, 33, 34. It is therefore natural to wonder whether a similar pattern exists between the local charges of the PCM on a supergroup and those of the affine Toda field theory on a Lie superalgebra [35, 36]. The difficulty here is that to each Lie superalgebra there may be associated more than one affine Toda field theory, depending on the choice of inequivalent simple root system. It would be interesting to consider whether the PCM shares any properties with these models.

Quantum conformal invariance is not expected for general Lie supergroups, but it should be possible to introduce a WZ term which guarantees this at a certain critical limit. The integrability of these models through a consideration of their local and non-local conserved charges will be the scope of future work.

Similar to the arguments in this paper, the sigma model with a supercoset target (either with or without a WZ term) should exhibit local conserved charges. The construction of a set of (classically) commuting charges for these models has yet to be explored.

For the PCM on a Lie Group in the presence of a boundary (i.e. on the halfline $x<0$ ) a natural connection arises between boundary integrability and symmetric spaces [37. Future work will investigate the analogous boundary conditions to ensure integrability on a supergroup.

Finally the particle spectrum of these models remains to be explored. Be- 
cause of the additional complications of the representation theory (i.e. atypicality [19]), this is expected to be more complicated for supergroups than for the bosonic models.

\section{Acknowledgements}

I would like to thank Niall MacKay for his advice and supervision, and Charles Young for useful discussions. I also thank EPSRC and the EUCLID network, ref. HPRN-CT-2002-00325, for funding.

\section{References}

[1] N. Berkovits, M. Bershadsky, T. Hauer, S. Zhukov, B. Zwiebach, Superstring Theory on $A d S_{2} \times S^{2}$ as a Coset Supermanifold, Nucl. Phys. B567 (2000) 61 hep-th/9907200

[2] I. Bena, J. Polchinski, R. Roiban, Hidden Symmetries of the $A d S_{5} \times S^{5}$ Superstring, Phys. Rev. D69 (2004) 046002 hep-th/0305116

[3] A. Das, J. Maharana, A. Melikyan, M. Sato, The Algebra of Transition Matrices for the $A d S_{5} \times S^{5}$ Superstring, JHEP 0412 (2004) 055 hep-th/0411200

[4] R. R. Metsaev, A. A. Tseytlin, Type IIB Superstring Action in AdS $S_{5} \times$ $S^{5}$ Background, Nucl. Phys. B 533 (1998) 109 hep-th/9805028

[5] C.A.S. Young, Non-local charges, $\mathbb{Z}_{m}$ Gradings and Coset Space Actions, Phys. Lett. B 632 (2006) 559 hep-th/0503008

[6] D. Kagan, C.A.S. Young, Conformal Sigma-Models on Supercoset Targets, hep-th/0512250

[7] N. Berkovits, C. Vafa, E. Witten, Conformal Field Theory of AdS Background with Ramond-Ramond Flux, JHEP 9903 (1999) 018 hep-th/9902098

[8] M. Bershadsky, S. Zhukov and A. Vaintrob, $P S l(n \mid n)$ sigma model as a conformal field theory, Nucl. Phys. B 559 (1999) 205 hep-th/9902180

[9] J.M. Evans, M. Hassan, N.J. MacKay and A.J. Mountain , Local conserved charges in principal chiral models, Nucl. Phys. B 561 (1999) 385 hep-th/9902008; Conserved Charges and Supersymmetry in Principal Chiral and WZW models, Nucl. Phys. B 580 (2000) 605 hep-th/0001222

[10] J.M. Evans, A.J. Mountain, Commuting Charges and Symmetric Spaces, Phys. Lett. B 483 (2000) 290 hep-th/0003264 
[11] J.M. Evans, D. Kagan, N.J. MacKay, C.A.S. Young, Quantum, Higherspin, Local Charges in Symmetric Space Sigma Models, JHEP 0501 (2005) 020 hep-th/0408244

[12] H. Saleur, B. Kaufmann, Integrable Quantum Field Theories with OSp $(m \mid 2 n) \quad$ Symmetries, Nucl. Phys. B628 (2002) 407 hep-th/0112095

[13] N. Mann, J. Polchinski, Bethe Ansatz for a Quantum Supercoset Sigma Model, Phys. Rev. D 72 (2005) 086002 hep-th/0508232

[14] P. Lavaud, Superpfaffian, math.GR/0402067

[15] Y.Y. Goldschmidt, E. Witten, Conservation Laws in Some TwoDimensional Models, Phys. Lett. B91 (1980) 392

[16] M. Hatsuda and K. Yoshida, Classical Integrability and Super Yangian on $A d S_{5} \times S^{5}$, hep-th/0407044

[17] R. Zhang, Representations of Super Yangians, J. Math. Phys. 36 (1995) 3854; The $g l(M \mid N)$ Super Yangian and its Finite Dimensional Representations, Lett. Math. Phys. 37 (1996) 419

[18] L. Dolan, C. Nappi, E. Witten, Yangian Symmetry in D=4 Superconformal Yang-Mills Theory, hep-th/0401243

[19] A. Frappat, A. Sciarrino and P. Sorba, Structure of Basic Lie Superalgebras and of their Affine Extensions, Commun. Math. Phys. 121 (1989) 457; Dictionary on Lie Algebras and Superalgebras, Academic Press, 2000

[20] V. G. Kac, Lie Superalgebras, Adv. in Maths. 26 (1977) 8

[21] Scheunert, Lie Superalgebras, Lecture Notes in Mathematics 716, Springer-Verlag, 1979

[22] J. A. Azcárraga, J. M. Izquierdo, A. M. Perelomov and J. C. Pŕez Bueno, The $Z_{2}$-graded Schouten-Nijenhuis bracket and generalised super-Poisson structures, J. Math. Phys. 38 (1997) 3735 hep-th/9612186

[23] L.D. Faddeev, L.A. Takhtajan, Hamiltonian Methods in the Theory of Solitons, Springer Series in Soviet Mathematics, 1987

[24] J. A. de Azcárraga, A. J. Macfarlane, A. J. Mountain and J. C. Pérez Bueno, Invariant tensors for simple groups, Nucl. Phys. B 510 (1998) 657 physics/9706006 
[25] A. J. Mountain, Invariant tensors and Casimir operators for simple compact Lie groups, J. Math. Phys. 39 (1998) 5601

[26] P. D. Jarvis, H. S. Green, Casimir invariants and characteristic identities for generators of the general linear, special linear and orthosymplectic graded Lie algebras, J. Math. Phys 20 (1979) 2115

[27] A. Sergeev, The invariant polynomials on simple Lie superalgebras, math.RT/9810111, An analog of the classical invariant theory for Lie superalgebras, math.RT/9810113

[28] E. Brézin, C. Itzykson, J. Zinn-Justin and J.B. Zuber, Remarks on the existence of non-local charges in two-dimensional methods, Phys. Lett. 82B (1979) 442

[29] N. J. MacKay, Introduction to Yangian symmetry in integrable field theory, Int. J. Mod. Phys. A 20 (2005) 7189 hep-th/0409183

[30] S. Parke, Absence of particle production and factorization of the $S$ matrix in 1+1-D models, Nucl. Phys. B174 (1980) 166

[31] G. Wilson, The Modified Lax and Two-Dimensional Toda Lattice Equations Associated with Simple Lie Algebras, Ergod. Th. Dynam. Sys. 1 (1981) 361

[32] D. Olive, N. Turok, Local Conserved Densities and Zero-Curvature Conditions for Toda Lattice Field Theories, Nucl. Phys. B257 (1985) 277

[33] E. Corrigan, Recent Developments in Affine Toda Quantum Field Theory, hep-th/9412213

[34] J.M. Evans, Integrable sigma-models and Drinfeld-Sokolov Hierarchies, Nucl. Phys. B 608 (2001) 591 hep-th/0101231

[35] J. Evans, J.O. Madsen, Dynkin Diagrams and Integrable Models based on Lie Superalgebras, Nucl. Phys. B503 (1997) 715 hep-th/9703065

[36] J. Evans, T. Hollowood, Supersymmetric Toda field theories, Nucl. Phys. B352 (1991) 723

[37] N.J. MacKay, B.J. Short, Boundary Scattering, Symmetric Spaces and the Principal Chiral Model on the Half-line, Commun. Math. Phys. 233 (2003) 313 hep-th/0104212 